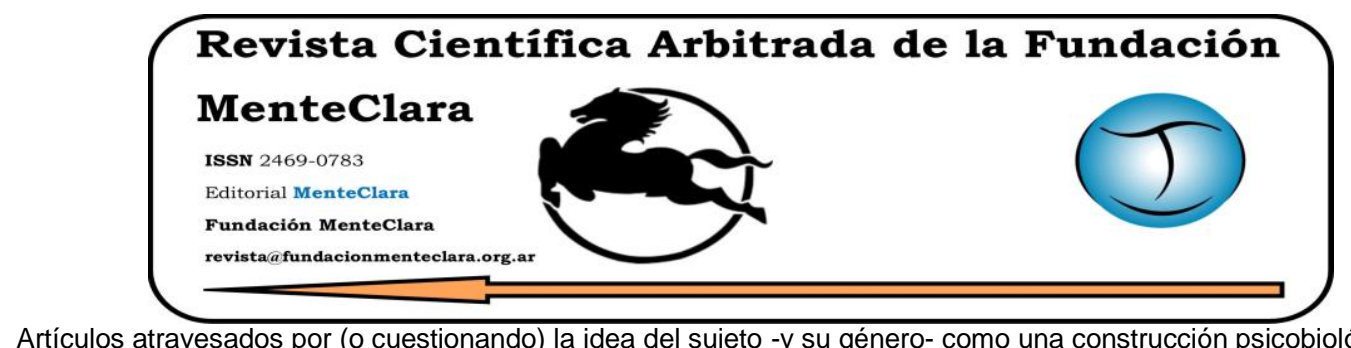

Artículos atravesados por (o cuestionando) la idea del sujeto -y su género- como una construcción psicobiológica de la cultura. Articles driven by (or questioning) the idea of the subject -and their gender- as a cultural psychobiological construction

Vol. 2 (2), 2017

ISSN 2469-0783

https://datahub.io/dataset/2017-2-2-e27

\title{
DESARROLLO Y ROL DE LAS PROVINCIAS. UN NUEVO ROL DE LOS ESTADOS PROVINCIALES EN MATERIA DE DESARROLLO ECONÓMICO.
}

\author{
DEVELOPMENT AND ROLE OF ARGENTINE PROVINCES. A NEW ROLE OF \\ PROVINCIAL GOVERNMENTS IN ECONOMIC DEVELOPMENT.
}

Hugo Daniel Guerrieri hdguerrieri@gmail.com

Facultad Latinoamericana de Ciencias Sociales. Sede Académica Argentina.

Cómo citar este artículo / Citation: Guerrieri H. (2017). "Desarrollo y rol de las provincias. Un nuevo rol de los estados provinciales en materia de desarrollo económico" Revista Científica Arbitrada de la Fundación MenteClara, 2(2), 143-168. DOI: $10.32351 /$ rca.v2.2.30

Copyright: (C) 2017 RCAFMC. Este artículo de acceso abierto es distribuido bajo los términos de la licencia Creative Commons Attribution-Non Commercial (by-cn) Spain 3.0. Recibido: 15/06/2017. Aceptado: 04/07/2017 Publicación online: $30 / 10 / 2017$

Conflicto de intereses: Ninguno que declarar.

\section{Resumen}

El objetivo general es formular un cambio de rol de los estados provinciales argentinos frente a los desafios que presenta el actual contexto internacional para nuestra Nación en materia de crecimiento sustentable y productivo basado en la equidad y la cohesión social con una perspectiva estratégica compartida. El estado nacional debe hacer uso de la racionalidad para poder planificar su desarrollo integral por lo que, frente a esta realidad, los estados provinciales deben reformular su rol para acompañar ese proceso con los suficientes recursos y con políticas e instrumentos verdaderamente efectivos para el desarrollo de las economías regionales. Tomamos como caso de estudio a la provincia de Buenos Aires y abordamos, a modo de ejemplo, el presupuesto provincial y el Régimen de Promoción Industrial, donde analizamos la distribución de los recursos y la aplicación del referido instrumento en los últimos 
cinco años y su impacto en la economía real bonaerense. Finalmente, formulamos nuestras ideas para el nuevo enfoque que proponemos, que apunta a producir un diametral cambio de dirección de la gestión provincial para promover con éxito el desarrollo económico.

\begin{abstract}
This article aims broadly at formulating a role change in Argentine provincial governments when tackling challenges presented by the current international context for Argentina in terms of sustainable and productive growth based on equity and social cohesion with a shared strategic perspective. The Argentine government must act rationally to be able to plan an integral development; accordingly, provincial governments must reformulate their role to accompany such process by using appropriate resources and applying truly effective policies and instruments for regional economies to develop. As a case study, we take the province of Buenos Aires' budget and its "Industrial Promotion Schedule", where we examine how resources were allocated and how said schedule was applied in the last five years as well as its impact on the real economy of Buenos Aires. Finally, we formulate our ideas for the new approach we propose, which aims at producing a dramatic change in the direction of provincial management for a successful economic development.
\end{abstract}

\title{
Palabras Claves/ Keywords
}

Desarrollo; Provincias; Rol. 


\section{Introducción}

Frente al desafio de la necesidad de un avance hacia un nuevo paradigma de desarrollo en el mundo en general y particularmente en la Argentina, y teniendo en cuenta que, para nosotros, el eje central es el aspecto económico, observamos que en las últimas décadas se fueron generando Ministerios de la Producción en los diferentes niveles del Estado y que, hasta el momento, carecen de los necesarios recursos presupuestarios, comparados con otras aéreas de la gestión pública.

Considerando que dichos Ministerios podrían cumplir un rol vital en lo que hace a la promoción del desarrollo en su aspecto económico, y que insistimos en señalar, que este aspecto debe ser la columna vertebral del desarrollo integral.

Mediante el presente estudio pretendemos proponer la posibilidad de un avance en las estructuras de los estados provinciales para estimular el desarrollo, como autentica manera de generar las condiciones para que las políticas e instrumentos en la materia se apliquen con el necesario éxito, a partir de contar con la necesaria capacidad de gestión.

Buscamos demostrar que si bien, muchas politicas e instrumentos pueden ser apropiados para promover el desarrollo económico, la mayoría de las veces, no tienen el debido alcance e impacto en las economias reales, quedando formulados solamente a nivel súper-estructural, por la falta de los recursos necesarios.

Partimos de considerar que el acento de las politicas públicas, a la hora de tomar decisiones de gobierno, es definido fundamentalmente por la proporción del presupuesto general que se le asigna. Y es en esta cuestión que vemos un retraso enorme en cuanto a lo que se viene determinando históricamente a las aéreas de producción en los presupuestos de las provincias. En ningún caso superan el 2\% del total presupuestario, 
implicando esta realidad el nivel de importancia que hasta ahora se le da a la temática, y por lo tanto el alto nivel de ausencia de los estados provinciales en el desarrollo de las economias regionales. Por lo que sostenemos que, en el camino de la construcción de un nuevo modelo de desarrollo, esta cuestión resulta determinante.

Abordamos, a modo de ejemplo, la provincia de Buenos Aires, ya que representa alrededor del 40\% de la economía real de la Argentina.

Analizamos su distribución presupuestaria en detalle para poder observar lo que se le asigna a la producción y estudiamos específicamente el caso relacionado con la aplicación del Régimen de Promoción Industrial por parte del Ministerio de la Producción, dado que se trata de un instrumento emblemático en materia de promoción del desarrollo económico en esta provincia.

De esta manera pretendemos cumplir, mediante este trabajo, con los siguientes objetivos:

1-Objetivo General: Formular un cambio de rol de los estados provinciales en materia de desarrollo económico.

\section{2-Objetivos Especificos:}

2-1-Demostrar la escases de recursos presupuestarios provinciales que se destinan a la producción.

2-2-Demostrar el impacto limitado de las políticas e instrumentos provinciales de promoción del desarrollo económico y la necesidad de mayores recursos. 
Estamos convencidos de que frente al desafio de avanzar hacia un nuevo paradigma del desarrollo debe existir un Estado Nacional que cumpla, entre muchos otros, ciertos roles fundamentales:

a) Generar un contexto macroeconómico lo más favorable posible, según las condiciones internacionales e intrínsecas del país.

b) Construir, junto a todos los actores de la comunidad Nacional un Plan Estratégico de Desarrollo.

c) Generar toda la infraestructura necesaria para facilitar el desarrollo.

d) Asegurar la generación y distribución de toda la energía que demande el referido desarrollo.

e) Colaborar con el crecimiento sostenido del mercado interno. En dicho marco, entonces la pregunta es; ¿Se deben reformular los roles de los estados provinciales para que, con politicas, instrumentos y los recursos necesarios cumplan, estos, un fuerte papel en la promoción efectiva del desarrollo de la economía real?

Para responder a dicha pregunta cumpliendo con los objetivos generales y específicos de este trabajo, primero profundizamos todos los conceptos que conforman los basamentos de esta investigación mediante la recuperación de los que entendemos como principales aportes y debates sobre el tema mediante el desarrollo del marco teórico.

En segundo lugar, como estudio de caso, abordamos el análisis de la distribución presupuestaria de la provincia de Buenos aires, para de esta manera, demostrar el poco financiamiento existente para las politicas de promoción económica. $\mathrm{Y}$, también, analizamos la instrumentación del régimen de Promoción industrial en esta provincia, en los últimos cinco años, a efectos de demostrar la poca efectividad de este instrumento, debido a los pocos recursos con que cuenta el Ministerio. 


\section{I-El Marco Teórico}

\section{-Nuevo Paradigma}

Teniendo en cuenta que cuando hablamos de paradigmas decimos que "considero a estos como realizaciones científicas universalmente reconocidas que, durante cierto tiempo, proporcionan modelos de problemas y soluciones a una comunidad científica" (Kuhn, 1962), como ya señalamos en la introducción, estamos convencidos de la necesidad de avanzar en la construcción y fortalecimiento del nuevo paradigma del desarrollo como única forma de lograr achicar la tremenda e injusta brecha de desigualdad social existente en el mundo y en la Argentina. Y considerando que un nuevo paradigma, a partir de este enfoque, generado en base a solidas fundamentaciones teórica, dará paso a la sumatoria de muchas experiencias empíricas que tiendan a modificar la realidad.

Si bien, se observa, en el presente, un nuevo avance de las políticas neoliberales en Latinoamérica, afirmamos que ello implica la necesidad de recuperación política de quienes sustentan las ideas vinculadas al nuevo paradigma del desarrollo y, a su vez, resulta menester una profundización de los conceptos teóricos que le den fuerza a nivel científico.

Como coincidimos en que el presente momento histórico requiere la elaboración de propuestas concretas que permitan generar cambios profundos, "este cambio épocal está en interrelación con la necesidad de reemplazar los enfoques teóricos hasta hoy disponibles en las Ciencias Sociales hacia otro paradigma (el productivo-inclusivo, proyecto nacional popular y neodesarrollista) que implica el cuestionamiento del paradigma dominante (el neoliberal)" (García Delgado- Ruiz del Ferrier, 2013: 67), entendemos que el camino hacia ese nuevo enfoque teórico implica un proceso, con avances y retrocesos, y es el aporte de ideas, conceptos y 
propuestas, lo que irá permitiendo la instalación de este en la realidad Argentina.

\section{-Desarrollo Económico}

Entendemos que el desarrollo debe ser integral, con calidad de empleo, con distribución de la riqueza, contemplando diferentes aspectos de la realidad, el político, el social, el económico, el tecnológico, el cultural, el humano, el institucional, entre otros.

Esto implica un determinado modelo de acumulación, "la acumulación intensiva del capital privilegia el cambio estructural liderado por el cambio técnico, elevada productividad, mayor equidad social, integración de la estructura productiva-social regional y la coevolución de las variables tecnológicas, económicas, sociales, institucionales, politicas y culturales" (Hernández, 1998: 47). Y, en el mismo sentido, cuando hablamos de desarrollo y sustentabilidad, coincidimos en que:

"Las cuestiones de volver sustentable el crecimiento a través de inversiones de capital que transformen la matriz productiva y exportadora hacia bienes y servicios de mayor valor agregado en tecnología y conocimiento, desarrollar sistemas nacionales de innovación, avanzar en un proceso de mejoramiento de la distribución del ingreso y mayor igualdad en el acceso a bienes materiales y espirituales, y construir una ciudadanía más soberana y autónoma, constituyen las metas a alcanzar si se trata de atravesar un verdadero proceso de desarrollo." (Schweinheim, 2011:2).

Entonces, queda claro que se puede hablar de Desarrollo Humano, Desarrollo Institucional, Desarrollo Cultural, Desarrollo con Inclusión, Desarrollo Sustentable, Desarrollo Sostenible, Desarrollo Tecnológico, Desarrollo Social...., pero si bien todos se encuentran interrelacionados, 
consideramos que la columna vertebral, de todos ellos, es el "desarrollo económico"

\section{-Rol del Estado}

Cuando abordamos el rol del estado en la materia, coincidimos en que:

"La globalización no ha cambiado la naturaleza del proceso de Desarrollo Económico. Este continúa descansando en la capacidad de cada país de participar en la creación y difusión de conocimientos y tecnologias y de incorporarlos en el conjunto de su actividad económica y de sus relaciones sociales. El Desarrollo Económico sigue siendo un proceso de transformación de la economia y de la sociedad en la acumulación de capital, conocimientos, tecnología, capacidad de gestión y organización de recursos, educación y capacidades de la fuerza del trabajo, y en la estabilidad y permeabilidad de las instituciones, dentro de las cuales la sociedad transa sus conflictos y moviliza su potencial de recursos. El desarrollo es acumulación en este sentido amplio y la acumulación se realiza, en primer lugar, dentro del espacio propio de cada país." (Ferrer, 1963: 360).

Afirmamos que debe existir un estado nacional fuerte que fundamentalmente, en primer lugar, actúe promoviendo el desarrollo económico, a partir de generar un contexto macroeconómico positivo, $\mathrm{y}$ de gestar un plan estratégico de desarrollo económico. Acerca de lo último estamos de acuerdo en que:

"Hablar de planificación significa fundamentalmente intentar responder la pregunta acerca de cómo harán nuestros paises para lograr un cambio estructural significativo que les permita 
acelerar y mejorar el proceso de desarrollo, revertir las relaciones asimétricas y ser más efectivos en la construcción de sus propios y peculiares destinos" (Sotelo Maciel, 2013: 50).

A nuestro entender, el tema de la planificación resulta vital, ya que la posibilidad de lograr un profundo acuerdo político, económico y social, entre todos los actores del ámbito nacional, en torno a metas, objetivos, prioridades y políticas, a corto, mediano y largo plazo, generaría condiciones para el país extraordinariamente positivas.

Seguramente que un contexto de esa naturaleza facilitaria el ingreso de inversiones de capital para la producción y, por otra parte, impactaría muy positivamente en las variables de la macroeconomía. Además el Estado, en sus tres niveles -Nacional, Provincial y Local-, debe llevar adelante con éxito politicas activas, mediante programas e instrumentos para, entre otras cosas:

a) Crear las condiciones necesarias para garantizar el crecimiento de las inversiones, ya que sin inversiones no hay crecimiento y sin este no es posible el desarrollo.

b) Estimular el desarrollo del valor agregado, tanto a nivel de manufacturas de origen Industrial -MOI- como de manufacturas de origen agropecuario - MOA-, ya que esta cuestión resulta vital en materia de generación de empleo y de riqueza.

c) Apoyar a las pequeñas y especialmente a las medianas empresas. Son este tipo de empresas las que además de ser importantes creadoras de trabajo, acompañan en su fortalecimiento el crecimiento de la economía.

d) Promover el desarrollo de las economias regionales, cuestión ligada al desarrollo territorial armónico y al crecimiento sectorial. 
e) Fortalecer a los distintos eslabones de las cadenas de valor, de tal manera de que todos los actores económicos traccionen hacia arriba.

f) Facilitar la creación de Clúster -Parques industriales sectoriales-, asegurando una mayor competitividad sistémica.

g) Colaborar con el avance de las empresas en investigación y desarrollo - I+D -, para acompañar la permanente evolución de los mercados a nivel tecnológico.

h) Asegurar la capacitación para la gestión de las empresas y para la especialización de la mano de obra, cuestión muy necesaria frente a la extraordinaria competencia actual de los mercados.

i) Facilitar el acceso al financiamiento de los actores económicos, de tal manera que todos cuenten con los recursos necesarios para acompañar el proceso de crecimiento.

Sobre la cuestión señalada, vale rescatar los siguientes conceptos:

"La tarea de construir el mercado igualando las oportunidades, mejorando las capacidades, desarrollando las instituciones y replanteando el papel de la empresa-equivale a crear un nuevo entorno para fortalecer el progreso económico, mejorar la distribución de los ingresos y generar empleo decente, en el que el factor de competencia sea la calidad de la mano de obra y no los bajos salarios. Las politicas públicas deben recurrir a instrumentos distintos de los utilizados en el pasado y actuar como catalizadoras de los procesos de transformación, respetando algunos requisitos básicos sin los cuales perderian efectividad. Ya hemos nombrado algunos. El primero de ellos es adoptar una estrategia económica de irrupción en el mercado mundial; el segundo, garantizar la continuidad en el tiempo de los programas; el tercero, lograr 
coordinación y consistencia con el resto de las políticas públicas. El cuarto consiste en crear instancias institucionales del Estado y de la sociedad civil que operen como contrapesos para reducir el riesgo de captura rentística, y el quinto, en que en su implementación impacte positivamente en la distribución de los ingresos." (Kosacoff, 2007: 93).

Para que todo esto se cumpla, debemos contar con una importante capacidad estatal en la materia, tanto de recursos, como de gestión de estos y principalmente además, de lidiar con los conflictos que surjan de los diferentes actores del ámbito productivo, "Si la planificación del desarrollo no aborda como desafio propio la construcción de capacidades estatales, no sólo en el plano de la provisión de bienes y servicios sino fundamentalmente en el terreno de la puja entre actores, no pasará de constituir una práctica superflua del proceso de desarrollo" (Sotelo Maciel, 2013).

\section{-Estados provinciales y recursos para el desarrollo}

En este caso, pretendemos aportar una mirada enfocada en el tema del rol de los estados provinciales en materia de promoción del desarrollo económico, vinculada a las disponibilidad de recursos de los presupuestos generales de las provincias, dado que siempre, cualquiera sea el enfoque acerca de las capacidades de los estados, la base resulta ser los recursos, tanto humanos, como materiales, coincidimos conceptualmente con que:

"El presupuesto constituye una herramienta que le permite a la entidad pública cumplir con la producción de bienes $y$ servicios para la satisfacción de las necesidades de la población de conformidad con el rol asignado al Estado en la economía. 
En este contexto, el presupuesto público moderno tiene dos elementos fundamentales: uno, relacionado con los objetivos $y$ los resultados esperados $y$, el otro, con la programación $y$ asignación de los recursos necesarios para el cumplimiento de dichos objetivo.” (Paredes, 2006: 37)

Observando que durante las últimas dos décadas se generaron dentro de las estructuras de los estados provinciales áreas de promoción del desarrollo económico -Ministerios de la Producción-, los que hasta ahora no cuentan con envergaduras importantes y considerando a este fenómeno como un proceso, es que proponemos que, necesariamente deben estos ministerios ir creciendo financiera y estructuralmente para gradualmente ir aumentando el impacto de sus politicas e instrumentos.

Y esta cuestión, para nosotros, resulta vital, debido a que adherimos al siguiente concepto: "el cambio de paradigma en la Argentina, con politicas de mayor intervención estatal a partir de estimulos al desarrollo de las capacidades productivas, de una administración estatal más atenta a la generación de puestos de trabajo y del financiamiento de centros tecnológicos de punta, debe asi revalorizarse y fortalecerse" (García Delgado- Ruiz del Ferrier, 2013: 74).

\section{II-Estudio de caso}

\section{-Análisis de los recursos destinados al desarrollo económico}

Como estudio de caso elegimos a la provincia de Buenos Aires porque, constituye, sin lugar a dudas, el principal distrito de la República Argentina. Con el 11\% del territorio nacional, es la más extensa del país, concentra el 39\% de la población y aporta el 37 \% del Producto Bruto Interno" (Panorama Productivo, 2013: 1), y por lo tanto resulta 
emblemática en cuanto a rol del estado en materia de desarrollo económico.

Esto implica, que esta provincia tiene una tremenda potencialidad económica, pero, más allá de los instrumentos y programas que se implementan para fomentar la economía bonaerense, no existe un rol preponderante del Estado en materia de desarrollo.

Esto lo explica su presupuesto, dado que si observamos lo proyectado para el corriente año (Ley 14.807, 2016), entre el Ministerio de Producción (0,2\%), el Ministerio de asuntos Agrarios (0,1\%) suman el 0,3\% del total. Es decir de un total de \$354.244.000.000- la provincia gasta en desarrollo solamente $\$$ 1.098.000.000- (Ver gráfico 1).

\section{GRÁFICO N 1}

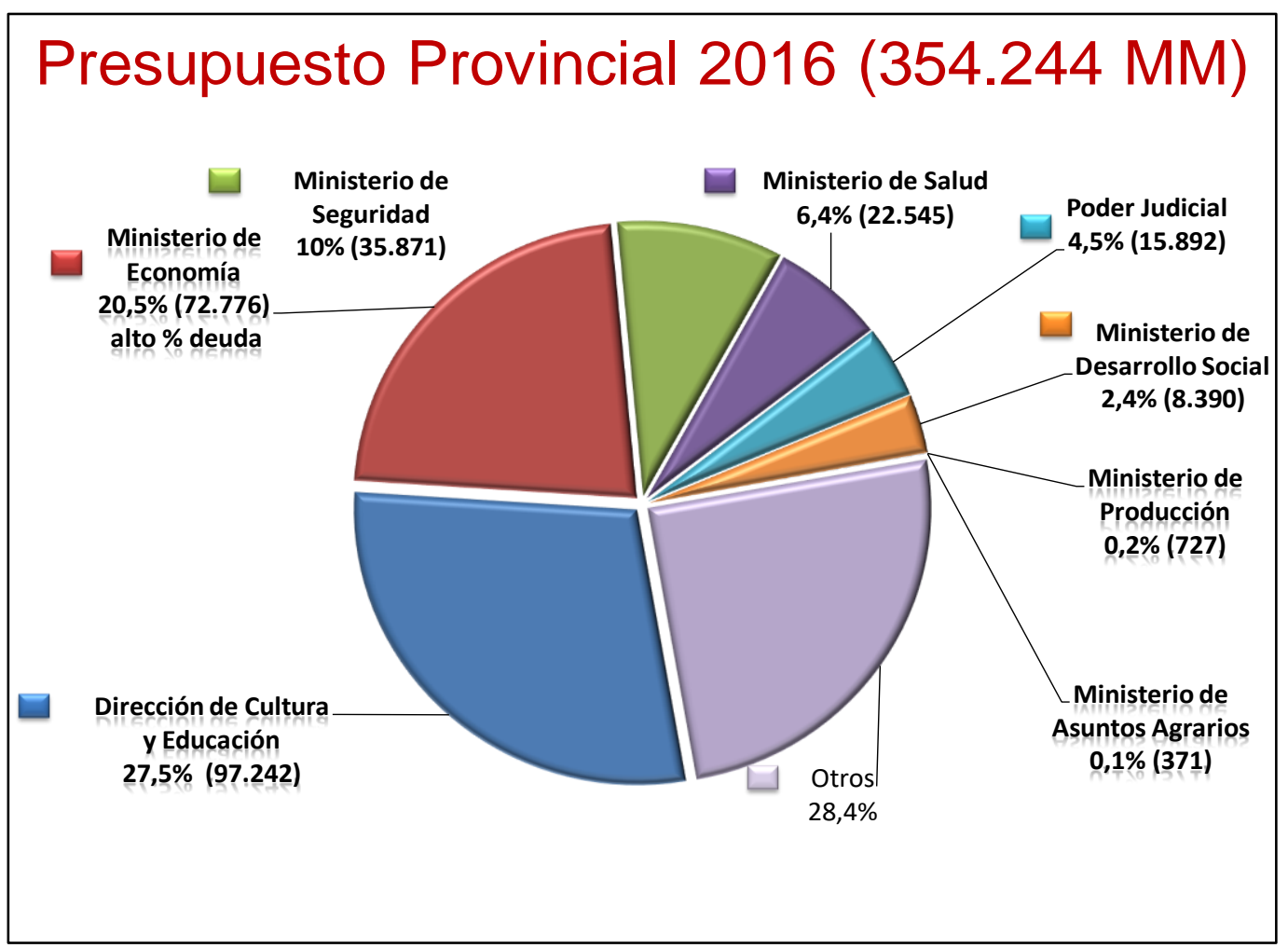

Fuente: Elaboración propia en base a Ley de Presupuesto 2016 
Claramente, en un presupuesto, donde se pone el dinero, se pone la decisión política, y esto significa que no hay política de desarrollo en la provincia de Buenos Aires.

Esta cuestión resulta histórica en la provincia, desde la creación de este Ministerio, se observan porcentajes similares de participación en el presupuesto general, es decir que siempre contó con fondos insuficientes como para cumplir un rol de importancia en la economía real de esta provincia. Por lo tanto se viene viviendo una inercia negativa caracterizada por el hecho de que año tras año ha crecido el endeudamiento público, y en la medida que aumentan las diferentes demandas de la sociedad en materia de seguridad, salud, educación, acción social, infraestructura, los recursos no alcanzan a dar las respuestas debidas, porque lo que recauda el estado es insuficiente dado que depende directamente del producto bruto interno y este no se expande según su potencialidad, debido a la ausencia, como ya señalamos, de un accionar importante del Estado.

Esta cuestión nos resulta de suma importancia, ya que se puede definir al presupuesto como la herramienta que le permite al sector público cumplir con la producción de bienes y servicios públicos para satisfacción de las necesidades de la población de conformidad con el rol asignado al Estado en la economía y sociedad del país.

La actividad presupuestaria del Estado, en cualquiera de sus niveles, se expresa como una asignación de recursos para el cumplimiento de una actividad concreta, en un periodo de tiempo, a fin de obtener unos resultados concretos -bienes y servicios- para la satisfacción de las necesidades públicas.

Existen por tanto, interrelaciones entre presupuesto, acción estatal y organización social. El presupuesto, en su esencia, antes que una 
herramienta jurídico-contable, constituye un instrumento de planificación y un componente importante de la política económica.

El desarrollo económico y social de las entidades públicas depende, en gran medida, de las decisiones que se tomen en materia de gasto público. El presupuesto público es una herramienta de politica gubernativa, mediante la cual se asignan recursos y se determinan gastos, para cubrir los objetivos. Y en el caso de las provincias, estos recursos son absolutamente necesarios para llevar adelante las politicas activas en pos del desarrollo económico.

\section{-Estudio del impacto de instrumentos provinciales de promoción del desarrollo}

Continuando con el análisis de la provincia de Buenos Aires, como estudio de caso, vemos que el Ministerio de la Producción de la Provincia de Buenos Aires es la autoridad de aplicación de la Ley de Promoción Industrial (Ley 13.656, 2007: articulo 30), marco normativo de fomento para la inversión productiva en la Provincia. Los beneficios otorgados por la Ley alientan la realización de inversiones promoviendo el desarrollo industrial y la incorporación de tecnologia en la industria con el objetivo de modernizar y tornar altamente competitivo al sistema productivo provincial

Este régimen de promoción tiene entre otros objetivos atraer inversiones productivas $\mathrm{y}$, en consecuencia, promover el desarrollo industrial de la provincia.

Los beneficios impositivos están orientados a estimular las nuevas inversiones productivas, el aumento de capacidad y la modernización de las empresas. Las empresas beneficiadas gozan de una exención total de hasta 10 años. Adhieren al Régimen de Promoción Industrial, las 
personas físicas residentes en el territorio provincial, las personas jurídicas constituidas en él o que se hallen habilitadas e inscriptas para actuar dentro de la provincia de Buenos Aires, cuyo propósito este orientado fundamentalmente al desarrollo de sus actividades productivas.

El artículo $1^{\circ}$ de la Ley que establece este régimen, tiene como finalidad:

a) Favorecer el desarrollo integral y armónico de la economía provincial.

b) Promover:

-El desarrollo industrial de la provincia a fin de consolidar su progreso económico con el objetivo de alcanzar el pleno empleo.

-La radicación industrial priorizando la descentralización económica con miras a afianzar núcleos de población y lograr un desarrollo geográfico equilibrado.

-La localización de industrias en los Agrupamientos Industriales aprobados por el Poder Ejecutivo Provincial.

-La radicación de empresas que brinden servicios a la industria y resulten de importancia relevante para su desarrollo.

c) Estimular:

-La formación de sistemas productivos regionales, constituidos por agrupaciones de empresas altamente especializadas, que tengan: proximidad geográfica, problemáticas productivas similares o complementarias y que se asocien para lograr ventajas competitivas. 
-El mejoramiento de la capacitación de la mano de obra con el objetivo de elevar la competitividad de la industria provincial.

-La vinculación de la industria con el sistema provincial de innovación tecnológica.

d) Ayudar a la preservación del medio ambiente y al uso racional de los recursos naturales.

e) Apoyar el desarrollo e incorporación de tecnología en la industria con el objetivo de modernizar y tornar altamente competitivo al sistema productivo provincial.

f) Propender:

-Al desarrollo del tejido productivo provincial mediante el incentivo a las pequeñas y medianas industrias a través de la facilitación del acceso a los beneficios de la presente ley con requerimientos procesales adecuados a las condiciones de las mismas.

-Al desarrollo de la industria provincial en consonancia con el interés general de la Nación.

g) Mejorar la competitividad de la industria provincial mediante la incorporación de tecnología y la adopción de modernos métodos de gestión.

Estas son las características principales de este régimen, y es debido a ellas, que lo elegimos como caso de estudio, dado que su finalidad cumple acabadamente con la idea de promoción del desarrollo económico.

Ciertamente que la inversión industrial, en los últimos cinco años, no ha sido la más relevante, comparada con otros momentos de la economía nacional, pero, en términos de desarrollo económico, la ampliación de la 
capacidad productiva, la innovación tecnológica, la modernización y la mejora de la competitividad de las empresas industriales configuran factores determinantes.

Por lo tanto, si hablamos de política de estado en esta materia, este régimen de promoción resulta ser un instrumento vital, y más si pensamos que en el momento que crezca la inversión, mayor será el impacto de este instrumento en la economía real de la provincia.

Luego de realizar diversas consultas con las autoridades del Ministerio de la Producción de la provincia de Buenos Aires y de analizar las características generales de los actores económicos bonaerenses, hemos llegado a la determinación de evaluar los siguientes aspectos cuantitativos y cualitativos de la aplicación del régimen durante los últimos cinco años:

a) Cantidad de industrias que iniciaron el trámite para acogerse al régimen.

b) cantidad de industrias que resultaron alcanzadas por el régimen.

c) Análisis de causas ante la brecha existente, entre ambos items anteriores.

d) La importancia de los beneficios que otorga este régimen.

e) La dimensión de la estructura del estado que se aboca a la aplicación de este régimen.

f) La asistencia a las industrias que realizan el trámite.

g) La difusión del régimen y sus beneficios.

h) La capacitación de las industrias de la provincia en relación al acceso al régimen. 
Por razones vinculadas con los alcances de este trabajo, solamente nos hemos limitado a relevar las opiniones, sobre los aspectos señalados, de dos grupos representativos: los integrantes del equipo de aplicación del régimen y de dos entidades industriales: ADIMRA -Asociación de Industriales Metalúrgicos de la República Argentina-, que es la única entidad gremial empresaria de segundo grado representativa de la industria metalúrgica en el ámbito nacional y reúne a 59 cámaras activas, tanto sectoriales como regionales y gran parte de estas son de la provincia de Buenos Aires y la Fundación Pro Tejer que defiende los intereses de toda la cadena de valor textil la que se destaca como actividad predominante en Mar del Plata, Pergamino y Luján.

Los resultados obtenidos, realizado el relevamiento de campo, demuestran el bajo nivel de impacto de este instrumento de promoción. Con relación a la cantidad de industrias que iniciaron el trámite para acogerse al régimen, el resultado es que, en los cinco años, un total de 328 industrias, (Gráfico $N^{\circ}$ 2), lo hicieron (82 en el 2011, 48 en el 2012, 70 en el 2013, 53 en el 2014 y 75 en el 2015).

\section{GRÁFICO N²}

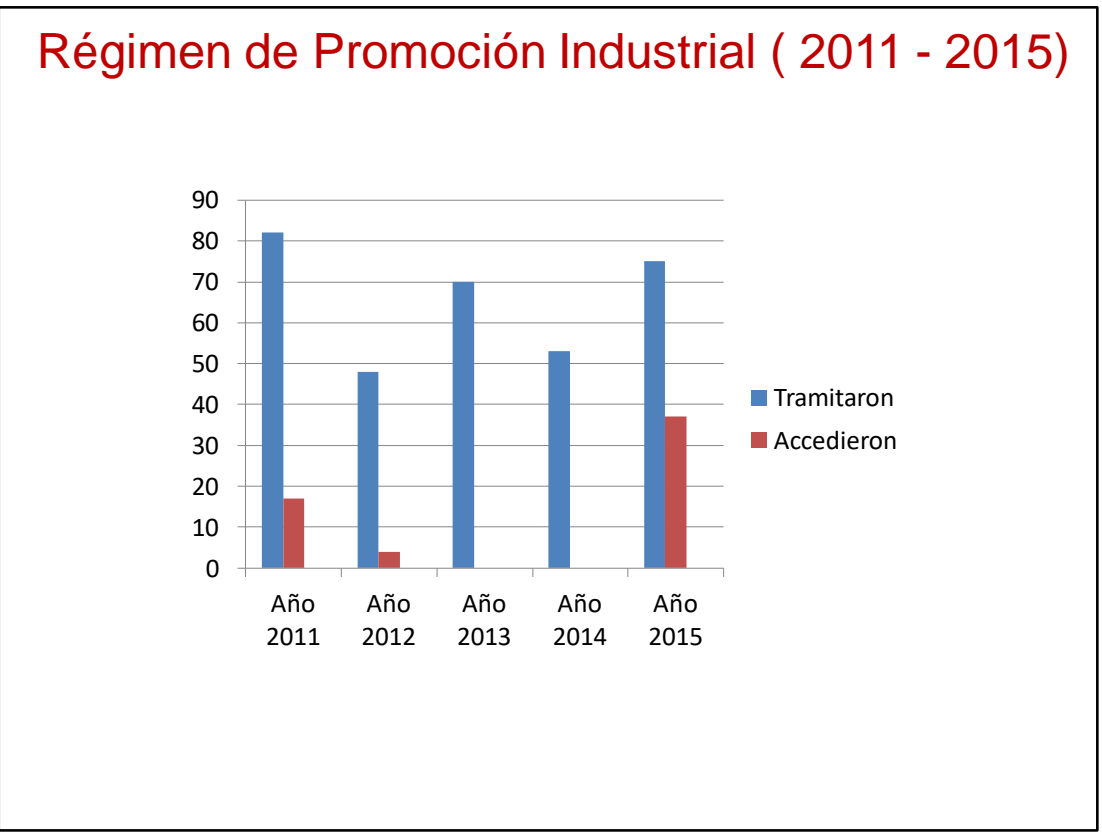

Fuente: Elaboración propia en base a relevamiento de campo 
Por otra parte, la cantidad de industrias que resultaron alcanzadas por el régimen, es decir que accedieron a los beneficios, durante ese mismo periodo, son 58 (17 en el 2011, 4 en el 2012, 0 en el 2013, 0 en el 2014, y 37 en el 2015). (Ver Gráfico $N^{\circ}$ 3).

Vemos que solamente pudieron acceder al régimen, el 17,68\% de las industrias que realizaron el trámite.

Para poder evaluar los alcances de la aplicación de este Régimen de Promoción Industrial, debemos analizar el universo de empresas que podrian acogerse al mismo.

Partiendo de un total estimado de alrededor de 28900 industrias radicadas en la provincia de Buenos y considerando que, si bien el nivel actual de inversión ha decrecido considerablemente, podemos afirmar que si solamente 58 industrias en cinco años accedieron, el impacto es verdaderamente infimo.

Al momento de indagar acerca de las principales causas observadas, por las cuales el resultado fue mínimo, se destacaron dos cuestiones:

a) Una importante demora por parte de las empresas en el cumplimiento de todos los requisitos formales del régimen,

b) Importantes restricciones presupuestarias del estado provincial.

Del relevamiento hecho, tanto en el estado, como en el sector privado, en cuanto a la importancia de los beneficios que otorga este régimen, podemos afirmar que se manifiesta cierto interés de parte de las industrias en que se mejoren los mismos. 


\section{GRÁFICO N³}

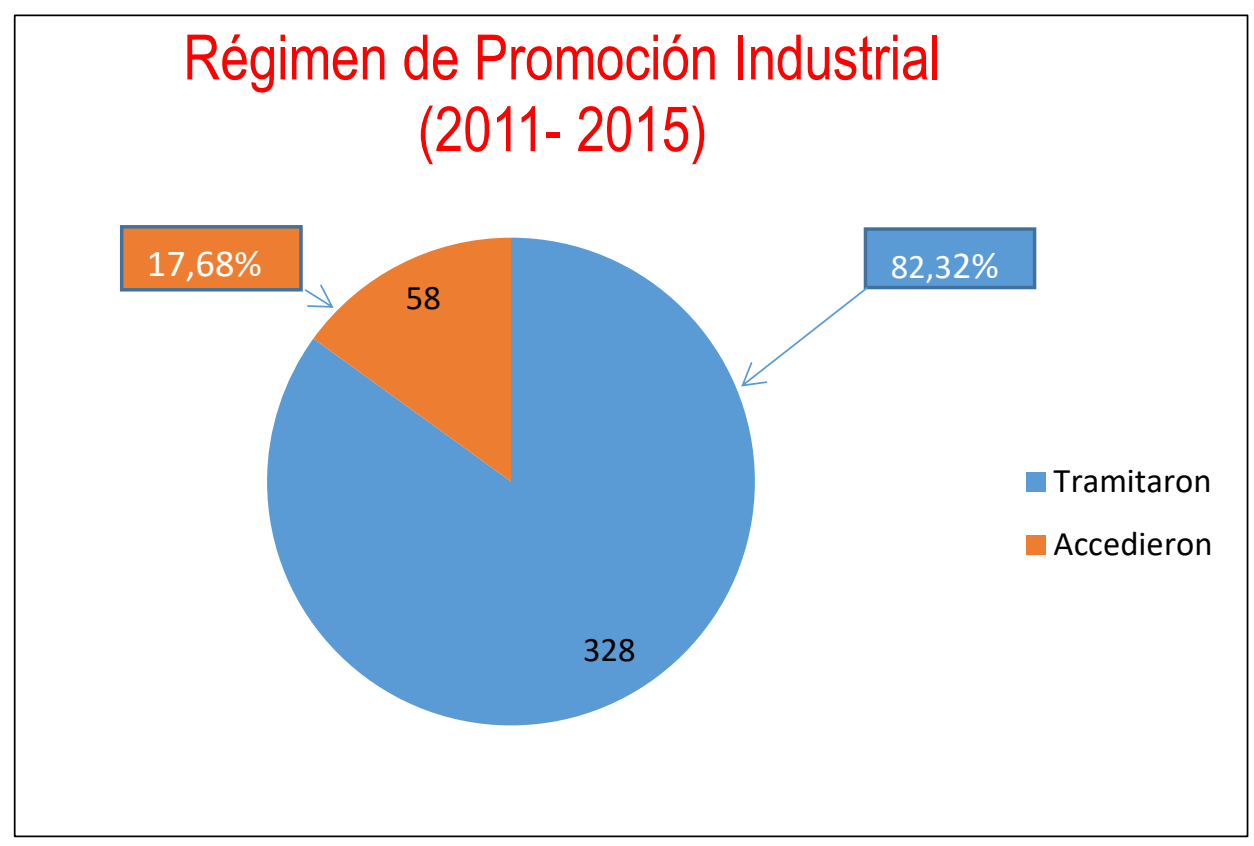

Fuente: Elaboración propia en base a relevamiento de campo

En cuanto a la dimensión de la estructura del estado que se aboca a la aplicación de este régimen, verdaderamente observamos que resulta muy reducida, teniendo en cuenta la gran extensión geográfica de la provincia y la cantidad y diversidad de industrias con proyectos de inversión radicadas, ya que cuenta solamente con un total de siete personas: un Director, un Subdirector, un Jefe de Departamento y 4 empleados.

Al analizar la asistencia que reciben las industrias que realizan el trámite, deducimos que, si bien la calidad de la misma es muy buena, evidentemente por lo señalado en cuanto a dificultad para completar los requisitos formales existe un déficit.

Por último en lo que hace a la difusión del régimen y sus beneficios, especialmente las cámaras, señalaron que no resulta suficiente ya que existen muchas industrias que desconocen al programa. 
Seguramente que también, para lograr un mayor cumplimiento de los requisitos del régimen por parte de las empresas, la capacitación de las industrias de la provincia resulta de suma importancia.

En cuanto a las causas del bajo impacto de este régimen podemos señalar, desde el lado empresario las siguientes:

a) Un marcado desconocimiento del régimen y de sus requerimientos.

b) Un déficit en materia de gestión para implementar la documentación necesaria para cumplir con los requisitos, especialmente en el sector Pyme.

c) Desconocimiento acerca de los beneficios y de sus alcances.

Y en relación con las causas relacionadas con el organismo de aplicación, observamos las siguientes:

a) La ausencia de suficiente personal para asesorar a las empresas, principalmente a las Pymes.

b) La falta de mayor difusión del régimen y sus beneficios.

c) Una reducida cantidad de evaluadores de los expedientes.

d) Una estructura demasiado limitada para atender a la potencial demanda, en el caso de que aumente la inversión. 


\section{III-Conclusiones y propuestas propias}

Queda claro que para que la aplicación de cualquier instrumento, política o programa del estado pueda llegar a impactar debidamente, se deben cumplir minimamente tres requisitos:

a) debe existir capacidad técnica y administrativa para asegurar una gestión eficaz y eficiente,

b) los cuadros de conducción del área deben tener la suficiente capacidad política para mediar frente a los diferentes conflictos de intereses, tanto de los actores económicos como de los diferentes estamentos burocráticos del propio gobierno,

c) se tiene que contar con los recursos financieros adecuados para asegurar la necesaria estructura de implementación y el suficiente sustento de los diferentes instrumentos de promoción.

Por lo tanto consideramos que, en este caso, si la provincia de Buenos Aires, quiere tener un estado verdaderamente promotor del desarrollo económico, el Ministerio de Producción deberá contar con un mayor presupuesto.

Es decir que el $0,2 \%$ del total del presupuesto, que dispone históricamente desde su creación, claramente no es suficiente como para producir un impacto valorable en la economía real bonaerense.

Entonces, sobre la base de que lo anteriormente concluido resulta igual en todas las provincias, nuestra propuesta consiste en revertir el enfoque existente diametralmente, otorgándole un rol importantísimo a los estados provinciales en lo que hace al desarrollo económico, a partir de ir destinando, paulatinamente, más partidas de los presupuestos generales y de reestructurar las áreas que comprenden a esta materia. 
Por ello, sostenemos que los presupuestos anuales deben reflejar las prioridades del gasto público, en este caso plasmadas en un Plan Provincial de Desarrollo.

Esta no es una tarea fácil, por cuanto requiere de un proceso coordinado de planeación a lo largo de todo el proceso presupuestal. De esa manera, se irá aumentando considerablemente la capacidad estatal de las provincias, de tal manera de lograr una verdadera promoción de la producción en todas las regiones y en todos los sectores económicos, y así asegurar un fuerte impacto positivo en la economía real.

Estimamos que deberá ser en forma progresiva, de tal manera que el crecimiento, en lo estructural, en el aumento de la capacidad de gestión politica y administrativa, y en lo que hace a la generación de nuevos instrumentos de promoción, determine con el tiempo que estas áreas de gobierno lleguen a cobrar una envergadura similar a las que hoy tienen suma relevancia.

Consideramos que esta propuesta permite revertir la inercia negativa existente -poco crecimiento, bajos recursos, mayor demanda social-, es decir, permite pasar a un positivo mayor crecimiento, más recursos, menos demanda social y más inclusión.

En la introducción decíamos:

"observamos que en las últimas décadas se fueron generando Ministerios de la Producción en los diferentes niveles del Estado y que, hasta el momento, carecen de los necesarios recursos presupuestarios, comparados con otras aéreas de la gestión pública. Considerando que dichos Ministerios podrian cumplir un rol vital en lo que hace a la promoción del desarrollo en su aspecto económico, y que insistimos en señalar, que este aspecto debe ser la columna vertebral del desarrollo integral". 
Continuando con esa línea afirmamos que ese proceso debe profundizarse cualitativa y cuantitativamente, de tal manera de transformarlos en Ministerios de Desarrollo Económico, cumpliendo, entre otras, las dos funciones claves:

a) generar los planes de desarrollo provinciales, concatenadamente con el Plan Estratégico de Desarrollo Nacional, y

b) la Promoción del Desarrollo Económico, mediante la gestión de las politicas, programas e instrumentos que determine el Plan.

Paulatinamente, estos ministerios deberían llegar a ser la columna vertebral de los gobiernos provinciales. Sin dudas, que este cambio diametral de enfoque propuesto, significaria un aporte importante a favor del nuevo paradigma del desarrollo. 


\section{Referencias}

Ferrer, A. (1963). La Economía Argentina. Desde sus origenes hasta principios del siglo XXI. Buenos Aires: Fondo de Cultura Económica.

García Delgado, D. y Nosetto, L. (2006) El Desarrollo en un nuevo contexto post-neoliberal. Hacia una sociedad para todos. Buenos Aires: Editorial Ciccus.

García Delgado, D. y Ruiz del Ferrier, C. (2013). El nuevo paradigma. Algunas reflexiones sobre el cambio épocal. Buenos Aires: Revista Estado y Políticas Públicas Nº1. FLACSO Argentina.

Hernández, R. D. (1998). Bases para un modelo de desarrollo, Tecnológico, Industrial y Regional. Buenos Aires: Ediciones Macchi.

Informe Especial, (2014). Acceso al financiamiento y crédito en PyME industriales. Buenos Aires: Fundación Observatorio Pyme.

Informe de Actualidad Industrial $\mathrm{N}^{\circ} 4$, (2016). Buenos Aires: Centro de Estudios de la Unión Industrial Argentina.

Kosacoff, B. (2007). Hacia un Nuevo Modelo Industrial. Idas y vueltas del Desarrollo Argentino. Buenos Aires: Claves para todos. Colección dirigida por José Nun. Capital Intelectual.

Kuhn, T. S. (1962). The Structure of Scientific Revolutions. Chicago: The University of Chicago Press [Trad. Esp. (1971). La Estructura de las Revoluciones Científicas, México: Fondo de Cultura Económica].

Ley 13.656, (2007). Régimen de Promoción Industrial de la provincia de Buenos Aires. Boletín Oficial de la provincia de Buenos Aires.

Ley 14.807, (2016). Presupuesto General de Gastos y Recursos de la Administración Provincial. Boletín Oficial de la provincia de Buenos Aires.

Ministerio de Economía de la provincia de Buenos Aires, (2013). Panorama productivo de la provincia de Buenos Aires. Dirección Provincial de Estudios y Proyecciones Económicas.

Paredes, F, (2006). Presupuesto público. Aspectos teóricos y prácticos. Venezuela: Publicaciones Vice-rectorado académico, Universidad de los Andes

Schweinheim, G. F. F. (2011). ¿Un nuevo desarrollo en América Latina? Implicancias en las políticas públicas, el Estado y la Administración. Caracas: Revista del CLAD (Centro Latinoamericano de Administración para el Desarrollo) Reforma y Democracia Nº49.

Sotelo Maciel, A. J. (2013). Planificación, desarrollo y capacidad política: Desafios de América Latina en el siglo XXI. Buenos Aires: Revista Estado y Politicas Públicas N 1 . FLACSO Argentina. 